\title{
Temperature determination via STJ optical spectroscopy
}

\author{
A. P. Reynolds ${ }^{1}$, J. H. J. de Bruijne ${ }^{1}$, M. A. C. Perryman ${ }^{1}$, A. Peacock ${ }^{1}$, and C. M. Bridge ${ }^{2}$ \\ 1 Research and Scientific Support Department of ESA, ESTEC, Postbus 299, 2200 AG Noordwijk, The Netherlands \\ 2 Mullard Space Science Laboratory, University College London, Holmbury St. Mary, Dorking, Surrey, RH5 6NT, UK
}

Received 11 December 2002 / Accepted 9 January 2003

\begin{abstract}
ESA's Superconducting Tunnel Junction (STJ) optical photon-counting camera (S-Cam2) incorporates an array of pixels with intrinsic energy sensitivity. Using the spectral fitting technique common in X-ray astronomy, we fit black bodies to nine stellar spectra, ranging from cool flare stars to hot white dwarfs. The measured temperatures are consistent with literature values at the expected level of accuracy based on the predicted gain stability of the instrument. Having also demonstrated that systematic effects due to count rate are likely to be small, we then proceed to apply the temperature determination method to four cataclysmic variable (CV) binary systems. In three cases we measure the temperature of the accretion stream, while in the fourth we measure the temperature of the white dwarf. The results are discussed in the context of existing CV results. We conclude by outlining the prospects for future versions of S-Cam.
\end{abstract}

Key words. instrumentation: detectors - stars: fundamental parameters - stars: novae, cataclysmic variables stars: white dwarfs - stars: individual: HU Aqr, EP Dra, UZ For, IY UMa

\section{Introduction}

The determination of astrophysical temperatures in the optical usually requires photometric filtering systems or dispersive optics. Recent developments in high-efficiency superconducting detectors, however, have opened the possibility of measuring photon energies directly (Perryman et al. 1993; Peacock et al. 1996). While the energy resolution possible with this technology is presently modest, it has already proven invaluable for studying faint, rapidly varying astrophysical sources such as cataclysmic variables. The study of colour ratio variations in these systems has already yielded significant new insights into the accretion regimes, the logical next step being to derive temperatures for the various system components.

ESA has developed two prototype cameras based on an array of STJ devices, and high time-resolution spectrallyresolved observations of rapidly variable sources such as cataclysmic variables and optical pulsars have been reported (Perryman et al. 1999; Perryman et al. 2001; Bridge et al. 2002a; Steeghs et al. 2002; Bridge et al. 2002b). In addition, we have now demonstrated that we can measure quasar redshifts directly with a precision of $\sim 1 \%$ (de Bruijne et al. 2002). The quasar study involved fitting a synthetic model to the observed distribution of photon energies in a given observation, and then adjusting the model redshift until a best-fit was obtained. In this paper we perform a similar study on a small sample of stellar observations. We demonstrate that we can fit black bodies to the data and thereby extract reasonable estimates of $T_{\text {eff }}$

Send offprint requests to: A. P. Reynolds,

e-mail: areynold@rssd.esa.int for objects spanning a range of temperatures between $\sim 3000$ $15000 \mathrm{~K}$. Having demonstrated the validity of the method, we then apply it to the specific case of accretion regions in several cataclysmic variable binary systems.

\section{Observations}

\subsection{The sample}

The sample of stellar observations consists of flux standards and scientific targets observed during three campaigns on the 4.2-m William Herschel Telescope on La Palma. Since these observations were conducted for reasons other than this study, the data set is rather inhomogeneous with regards to neutral density filter settings, seeing conditions and $\mathrm{S} / \mathrm{N}$. The observations are listed in Table 1. Spectral types are assigned on the basis of the current SIMBAD entry, while $T_{\text {eff }}$ values are derived from the literature. The hot white dwarf G191-B2B was used to constrain the instrumental response at high energies and is therefore omitted from the subsequent discussion.

\subsection{The instrument}

S-Cam2 is ESA's second STJ camera and was used on the three campaigns during which the observations were made. The instrument was mounted at the Nasmyth focus of the WHT during all the three campaigns. The camera contains a $6 \times 6$ staggered array of $25 \times 25 \mu \mathrm{m}^{2}\left(0.6 \times 0.6 \operatorname{arcsec}^{2}\right)$ tantalum junctions. The arrival times of individual photons are recorded with an accuracy of $5 \mu$ s, with an energy resolving power $R \sim 8$. 
Table 1. Stellar targets for temperature determination: literature values and S-Cam2 results. The column headed ND indicates the neutral density filter setting. Upper and lower values for $T_{\text {eff }}$ (Obs) show temperature extremes corresponding to gain variations of $\pm 1.0 \%$.

\begin{tabular}{rcrlllll}
\hline \hline No & Date & Exposure (s) & ND & Star & Type (Lit) & $T_{\text {eff }}(\mathrm{Lit} ; \mathrm{K})$ & $T_{\text {eff }}($ Obs; K) \\
\hline 1 & $1999-12-09$ & 600 & 3 & AD Leo & M3.5V & 3400 & $3100_{3000}^{3300}$ \\
2 & $1999-12-10$ & 1320 & - & G117-B15A & DA & $11500-12620$ & $13400_{11200}^{16300}$ \\
3 & $1999-12-16$ & 900 & 2 & G191-B2B & DAw & 56000 & - \\
4 & $1999-12-16$ & 1200 & 3 & Feige 15 & A0 & 10800 & $10400_{99100}^{12,800}$ \\
5 & $1999-12-16$ & 1800 & 2 & EV Lac & M3.5 & 3300 & $3200_{3100}^{3500}$ \\
6 & $1999-12-16$ & 900 & 2 & Feige 25 & B7 & 12800 & $11400_{9950}^{14,100}$ \\
7 & $2000-04-27$ & 600 & - & G138-31 & DA8 & $6300-6870$ & $7200_{6600}^{8100}$ \\
8 & $2000-04-28$ & 600 & 1 & HZ 21 & DA & 48000 & $100000_{400000}^{1000000}$ \\
9 & $2000-04-28$ & 600 & - & GD337 & DA+ . & $9250-15000$ & $11300_{9900}^{13600}$ \\
10 & $2000-09-29$ & 300 & 3 & BD +284211 & Op & 82000 & $44900_{200000}^{100,000}$ \\
\hline
\end{tabular}

1: Legget et al. (1996).

2: Robinson et al. (1995) favour 12375 K; Bergeron et al. (1995) favour 12620 K; Fontaine et al. (1996) favour 11500 K; mean value (12 $165 \mathrm{~K})$ plotted in Fig. 2.

3: Wolff et al. (1998), used for calibration purposes in this paper.

4: $T_{\text {eff }}$ estimated from A0 spectral type (Zombeck 1990).

5: Amado \& Byrne (1997).

6: Keenan \& Dufton (1983).

7: Greenstein (1984) gives DA8, which implies $T_{\text {eff }}=6300 \mathrm{~K}$ (Sion et al. 1983); Bergeron et al. (2001) give $T_{\text {eff }}=6870 \mathrm{~K}$; mean value (6585 K) plotted in Fig. 2.

8: Koester et al. (1979).

9: $T_{\text {eff }}=9250 \mathrm{~K}$ derived by authors using $G-I$ data and $G-I$ versus $T_{\text {eff }}$ relationship in Greenstein (1976); $T_{\text {eff }}=15000 \mathrm{~K}$

from $G-R$ colours in Greenstein (1975), using $G-R$ versus absolute magnitude $\left(M_{1.85}\right)$ and $M_{1.85}$ versus $T_{\text {eff }}$

calibrations in Greenstein (1976); mean value (12125 K) plotted in Fig. 2.

10: Herbig (1999).

The wavelength response of the instrument is $310-720 \mathrm{~nm}$. The violet cutoff is determined by the atmospheric absorption window, while the red cutoff is caused by the presence of long-wavelength filters designed to reduce thermal noise. Observations were made in conditions ranging from good to poor seeing, and with airmasses in the range 1.05-1.54.

\section{Data reduction}

Although we are dealing with optical spectra, our techniques and tools are those of high-energy astrophysics and we therefore find it more natural to discuss our data in the energy domain. We are typically dealing with photons in the range $\sim 1.5-$ $4 \mathrm{eV}$, about a thousand times less energetic than soft $\mathrm{X}$-ray energies.

The output from a single S-Cam2 observation consists of a list of photon arrival times, together with the pixel in which each event was recorded and the channel in which the event has been assigned by the pulse height analyser (PHA) electronics. The assigned channel depends linearly on the charge released by each photon when it arrived, which in turn depends linearly on the energy of the photon. The overall relationship between photon energy and channel number is therefore given by $E_{i}=$ $G \cdot E_{\mathrm{p}}+C$, where $E_{\mathrm{p}}$ is the energy of the incident photon in $\mathrm{eV}$, and where $G$ and $C$ are the gain (in channels/eV) and offset (in channels) of the relevant pixel. The gain and offset properties of all pixels have been mapped and found to be linear and stable for a given temperature of the detector holder. However small variations in the detector holder temperature and the electronic environment of the array can cause variations in gain of $\leq 1 \%$ during the course of a set of observations. This gain "jitter" cannot be determined independently of the observations and therefore sets a limit on the accuracy with which temperatures can presently be determined.

Data reduction proceeds as follows. We first select the time interval of data which is to be used to generate an output spectrum. If necessary, spatial filtering may also be applied at this stage by selecting and deselecting pixels, e.g., utilising only the pixels which see sky rather than source. For each selected pixel we then adjust the counts in each PHA channel to remove known analogue-to-digital artifacts. This results in a PHA spectrum at the particular gain and offset of that pixel. We then resample this spectrum such that it is on a common gain scale corresponding to a well-behaved reference pixel, and then coadd the contributions from all selected pixels, making an appropriate correction for the small differences in detection efficiency from pixel to pixel. The gain and offset of the reference pixel are assumed to be $G=42.5$ channels $\mathrm{eV}^{-1}$, and $C=$ -2.0 channels.

The extracted spectra will also contain a contribution from the sky background which - depending on the source brightness - may need to be removed before model fitting can take place. The sky contribution can be estimated in two ways. If the seeing was good, then the corner pixels of the array may be 

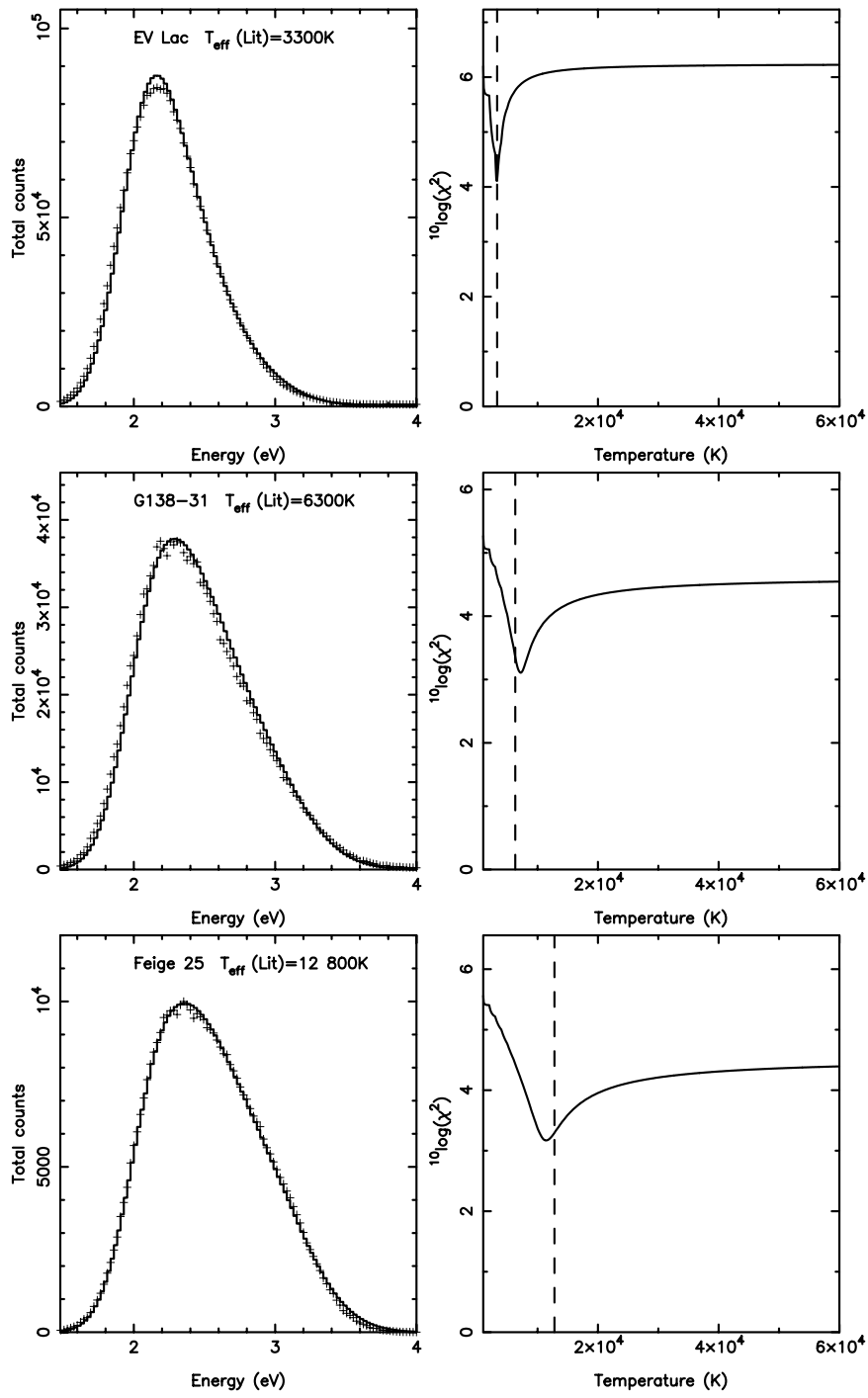

Fig. 1. Results for EV Lac, G138-31 and Feige 25. Left: the model fits to the PHA data (data points indicated by crosses; model curves by histograms). The fits are shown against energy rather than channel number for clarity. Right: the dependence of the fit statistic $\chi^{2}$ (plotted logarithmically) with temperature $T$. Vertical dashed lines indicate the literature values of $T_{\text {eff }}$.

used to obtain a sky spectrum. The advantage of this approach is that it ensures that the sky brightness is appropriate for the observation in question. The second approach is to use a sky frame obtained close (in time and position) to the observation in question. This approach has the advantage that many pixels are being sampled for the sky, smoothing out variations in gain away from the nominal values. In the case of this study we used corner pixels when the seeing was good, but when this was not the case we relied on sky frames taken as close as possible to the observation. These were not dedicated sky frames, but the results are relatively insensitive to the details of the background subtraction; indeed for most of the objects in our sample the background contribution is small enough to have little effect on the measured temperature.

\section{Temperature determinations}

\subsection{Method}

We discuss two complimentary approaches to temperature determination. In both cases we compare model spectra with the observed distribution of photon energies, searching for the closest match. The first approach uses synthetic black body spectra (Sect. 4.2), while the second uses a library of stellar templates of varying spectral type (Sect. 4.3).

In both cases due allowance is made for the finite energy resolution of S-Cam2, the transmissive properties of the atmosphere, telescope optics and detector elements, and the overall quantum efficiency of the detector. We make no allowance for the reddening effects of circumstellar and/or interstellar extinction, although our results suggest that this is not a serious drawback, at least in relation to our current sample of stars. For the model fitting, use is made of the XSPEC spectral analysis package (Arnaud 1996), a well-known tool for X-ray astronomy, but which is equally suited to the analysis of low-energyresolution optical data. Apart from the data (including target and background files), the two inputs that XSPEC requires are a response matrix and a model spectrum with one or more adjustable parameters. The response matrix specifies the transformation of the model spectrum from infinite energy resolution to the degraded energy resolution of the device, with the folding in of the overall detector efficiency. XSPEC then finds the set of model parameters that result in the lowest value of $\chi^{2}$. The package contains a large number of internal models (including a black body) but these were designed for the analysis of satellite data and are not modified for the effects of atmospheric absorption. We therefore supply our own models, adjusted for the mean atmospheric transmission corresponding to the specific airmass of each stellar observation. We created a grid of models spanning temperatures between 1000 and $100000 \mathrm{~K}$, and then used XSPEC to find the temperature corresponding to the best-fit black body, interpolating between grid values.

\subsection{Results: Black body fits}

In Fig. 1 we show the data and model fits for three of our observations, together with the corresponding curves of temperature versus $\chi^{2}$. Data channels 61-179 are included in the fits, corresponding to the $\lambda \sim 310-720 \mathrm{~nm}$ bandpass of the instrument, plus an additional 10 channels at the red and blue ends to account for the overspill of detected events due to the finite energy resolution.

The models are the input black bodies after modification for atmospheric transmission and convolution with the overall detector response. As is clear from the minima in the adjacent $\chi^{2}$ versus $T$ plots, the fits are not formally acceptable, for which one would expect $\chi^{2}$ in the range 50-150. Nonetheless there are well-defined minima in the $\chi^{2}$ versus $T$ curves, indicating the validity of the fitting process. These are all stars with literature $T_{\text {eff }}<15000 \mathrm{~K}$. The two hottest stars in our sample both have $T_{\text {eff }}>40000 \mathrm{~K}$, and in these cases there is no minimum in the $\chi^{2}$ versus $T$ curve, since for these objects we are only 


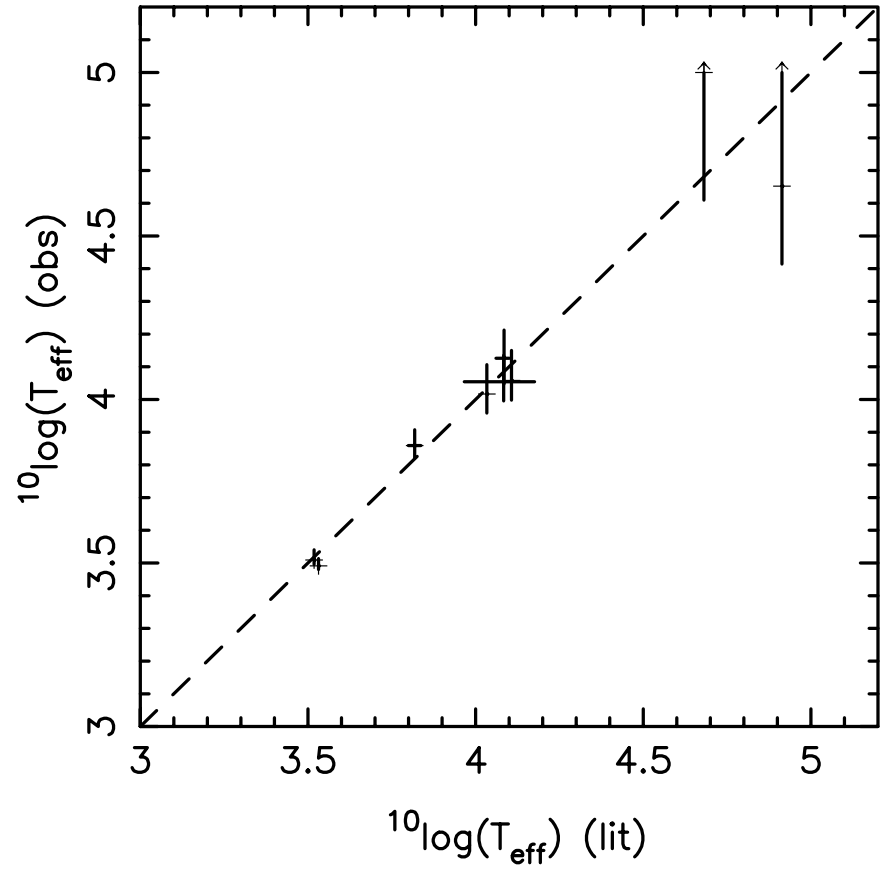

Fig. 2. Observed $T_{\text {eff }}$ versus literature temperatures for the nine stellar objects in our sample. The vertical error bars indicate the extreme range of temperatures corresponding to variations in the gain by $\pm 1 \%$. The dashed line shows the nominal 1:1 correlation.

fitting the red tail of the black body curve, which varies only slowly for large increases in temperature.

In Fig. 2 we show the observed versus literature temperatures for the nine stars in our sample. Horizontal error bars on some points indicate published uncertainty in the literature temperatures. Where there are two or more literature values we use a mean value (see Table 1 for notes on literature temperatures and adopted means). The vertical error bars reflect the uncertainty in the gain at the time of the observation, which is typically much larger than the uncertainty introduced solely by the fitting process (but see Sect. 5). We estimate the gain uncertainty in the following fashion.

It is known that small $(\mathrm{mK})$ variations in the temperature of the detector holder in the S-Cam2 cryogenic system lead to short-term variations in the gain on a timescale of minutes to hours, but the instantaneous gain can presently only be measured directly when observations are not in progress. This is done using an internal calibration source consisting of a red LED which illuminates the whole array. Several LED frames were obtained most nights, with $\sim 10-15$ frames per campaign. An analysis of the LED data showed that the gain varied from one LED frame to another with a standard deviation $(1 \sigma)$ of 0.4 channels $\mathrm{eV}^{-1}$, or $\sim 1 \%$ of the gain. If this gain "jitter" is also applicable to the observation data set, we should expect that $\sim 67 \%$, or $\sim 6$, of our observations, should have gains that lie within 0.4 channels $\mathrm{eV}^{-1}$ of the nominal gain of 42.5 channels $\mathrm{eV}^{-1}$. All else being correct, therefore, $\sim 6$ of our observations should have error bars that intersect the diagonal relationship between $T_{\text {eff }}$ (literature) and $T_{\text {eff }}$ (observed), with $\sim 2-3$ outliers. To investigate whether or not this is the case, we therefore regenerated the response matrix for $\pm 1.0 \sigma$ extreme gain ranges and remeasured the data using the black body fits. The resultant temperatures for the upper and lower gain cases are indicated by the vertical error bars. As is evident from Fig. 2, seven targets have error bars that intersect the diagonal, with only two outliers, one of which has rather an uncertain literature temperature. We therefore conclude that the only significant source of error in our analysis is due to the uncertain gain variations, and that this is entirely consistent with the gain jitter as characterised by the LED frames. Note that for the two hot stars in our sample, the black body fits reach the $100000 \mathrm{~K}$ model limit at the central gain and/or the lower gain limit, and so the error bars are only constrained at the lower temperature range.

\subsection{Results: Stellar template models}

The results shown in Fig. 2 suggest that the method works at least as well for cool stars (M-types) as it does for the hotter objects, and that there is no significant source of error beyond the gain uncertainty. Nonetheless, while spectra of hot white dwarfs do indeed resemble black bodies, this is not the case for the line-dominated cooler stars. To investigate whether the use of realistic spectra would improve the determinations, we made use of a library of digital spectra obtained at Kitt Peak National Observatory (Jacoby et al. 1984). The library contains 161 stars with spectral types between $\mathrm{M}$ and $\mathrm{O}$. We selected 20 stars of luminosity class $\mathrm{V}$, with spectral types between M5V and $05 \mathrm{~V}$. The digital spectra were then used as input models in the spectral fitting process, again folding in atmospheric absorption. In this case we simply selected the template that gave the lowest $\chi^{2}$ against an observation.

The resultant minimum determines the spectral type of the observation, from which an estimate of the temperature can be made. This is of course a less direct determination of temperature than black body fitting. Given the relatively small number of objects in our template selection, we estimate that the precision of the spectral class assignment is $\simeq 2$ subclasses. Here we discuss only the non-degenerate stars in our sample. AD Leo and EV Lac are both cool M3.5 flare stars, while Feige 15 is an A0 star. For AD Leo and EV Lac we determine the spectral type to be M5 in both cases, slightly cooler than the true spectral type of M3.5. For Feige 15 we find a spectral type of B6, somewhat hotter than the true value of A0. The results are therefore acceptable within the anticipated error, but show no obvious improvement in comparison with the black body estimates. Improved results might be obtained with a larger set of templates, but for the time being there is no clear disadvantage in using the pure black body models, even for cooler stars.

\subsection{Spectral distortion due to pile-up}

In common with all photon counting detectors, S-Cam2 has a finite response time associated with the arrival of a single photon. If a second photon arrives during this interval, the detection of one or both may be affected. Although a naive consideration might suggest that two photons arriving within a short time would always lead to a higher energy being detected, in 
practice the detailed properties of the electronic shaping mean that possible outcomes can include not only the assigning of higher energies to one or more events, but also the assigning of lower energies or even the non-detection of an event. The net result is that as count rates increase, recorded spectra suffer increasing distortion, with events being redistributed to both higher and lower energy channels.

The electronics of S-Cam2 are such that only photons arriving on a single pixel within $\sim 200 \mu$ s of a preceding photon will be affected by pile-up. Pile-up should therefore be of little consequence for count rates below $500-1000 \mathrm{~Hz}$ per pixel, a prediction which has been verified in the laboratory. Since the maximum count rates of our stellar observations are all $<800 \mathrm{~Hz}$ per pixel, pile-up is not expected to cause significant error in the derived temperatures. To explore this further, we have simulated the electronic response of S-Cam2 to the rapidly varying intensity profile of the Crab pulsar, an object that was observed during several S-Cam campaigns (Perryman et al. 1999). The instantaneous intensity of the Crab pulsar and its background nebula component ranges from $\sim 100$ counts $\mathrm{s}^{-1}$ per pixel in the interpulse to $>2000$ counts $\mathrm{s}^{-1}$ per pixel during the main pulse. Our phase-resolved observations of the Crab were split into three energy bands, corresponding roughly to red $(R)$, visual $(V)$ and blue $(B)$. From these three energy bands we construct two colour ratios: $V / R$ and $B / V$, and plot them against phase (Fig. 3). Significant variations are apparent, especially in the $B / V$ data, but after dividing out the predicted variations due to pile-up, there is no longer any strong evidence for colour changes across the pulse profile. This is consistent with previous studies in the UV-optical-IR band, which have reported either no colour changes (e.g., Carramiñana et al. 2000), or very slight ones (e.g., Eikenberry et al. 1996; Romani et al. 2001). This suggests that the pile-up characteristics of S-Cam2 are well understood, at least in the count rate regime probed by the Crab.

Using the same approach, we have created a synthetic input stellar spectrum and subjected this to simulated pile-up distortion for count rates ranging from 10-3000 counts $\mathrm{s}^{-1}$ per pixel. At each count rate we determine the temperature via the same black body fitting method outlined earlier. We generated ten simulations at each count rate step, measuring the temperatures individually and deriving their mean and variance.

The results, shown in Fig. 4, indicate that pile-up has no significant effect on the measured temperature for count rates below $\sim 250$ counts $\mathrm{s}^{-1}$ per pixel, and is modest for count rates $\leq 750$ counts $\mathrm{s}^{-1}$ per pixel. Once the count rate exceeds $\sim 1000$ counts $\mathrm{s}^{-1}$, however, the distortion becomes severe and pile-up rivals gain uncertainty as the main source of error in temperature determination. Similar conclusions are drawn using a hotter input spectrum. None of the stellar observations discussed in this paper had individual pixel count rates in excess of 700 counts $s^{-1}$, indicating that pile-up is not the major source of error in our data. In future versions of the instrument, however, where the gain variations are likely to be better constrained, an accurate treatment of pile-up may be necessary in order to extract the most accurate temperature determinations.

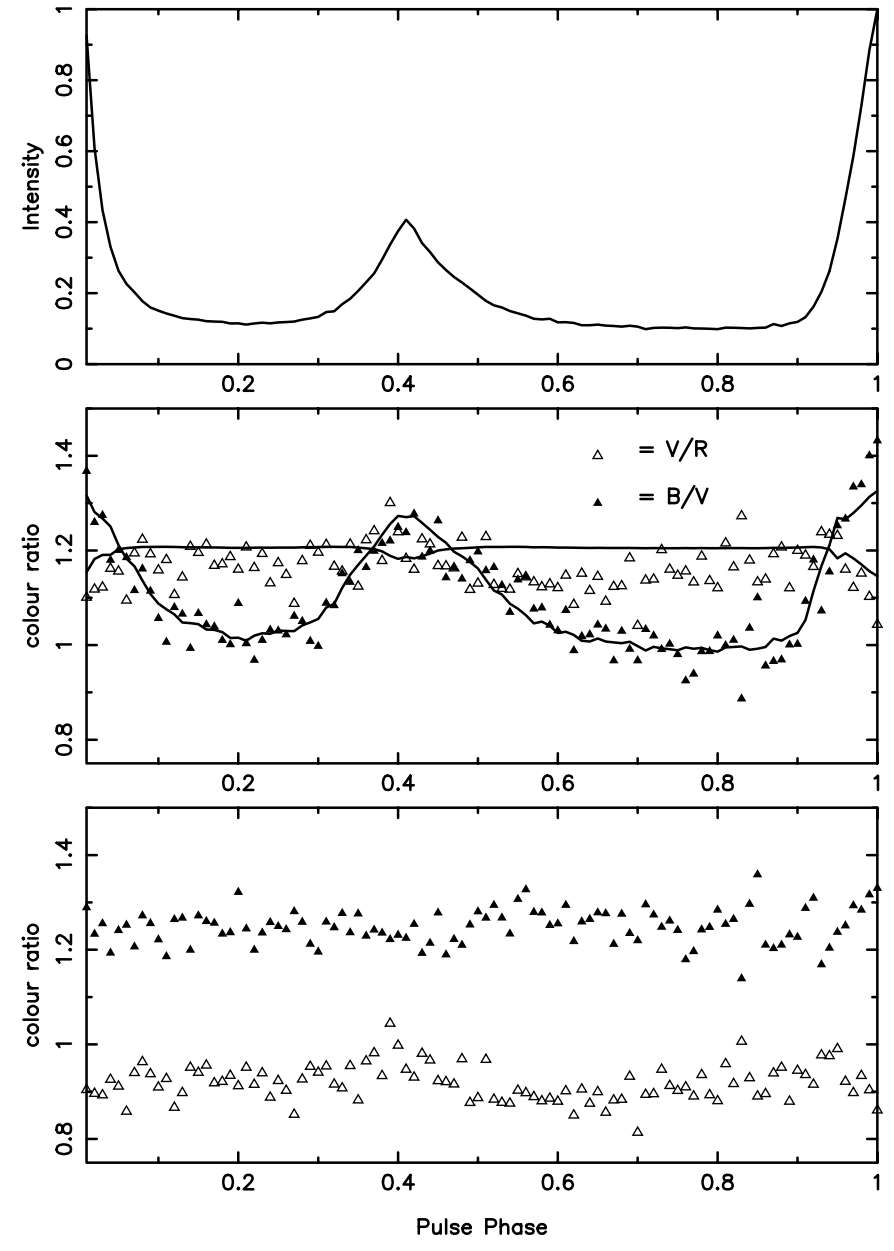

Fig. 3. Colour ratios for the optical light curve of the Crab pulsar. Top: the observed intensity profile of the Crab. Middle: the measured $V / R$ and $B / V$ colour ratios (symbols) and the predicted variations for the same ratios (lines). Bottom: the measured colour ratios after dividing by the predicted variations. Arbitrary offsets have been applied for the sake of clarity.

\section{Application of the technique: Cataclysmic variable stars}

During the course of the three S-Cam2 campaigns we also made a number of observations of eclipsing cataclysmic variables (CVs). Three systems were polars and one a dwarf nova. Reports on the S-Cam2 observations of the polars UZ For, HU Aqr and EP Dra may be found in Perryman et al. (2001), and Bridge et al. (2002a, 2002b). S-Cam2 observations of the dwarf nova IY UMa are reported in Steeghs et al. (2002).

The main difference between these systems is in the accretion process, which is in turn determined by the strength of the magnetic field. In polars the field is sufficiently strong that the accretion stream from the secondary becomes magnetically confined in the vicinity of the dwarf. From this point accretion material follows magnetic field lines to the dwarf surface, where accretion occurs through a stand-off shock. In the dwarf novae, the magnetic field is weak or absent, such that accretion is via a disk.

Prior to the eclipse, the dominant source of optical emission is the central accretion region, be it the white dwarf, one 


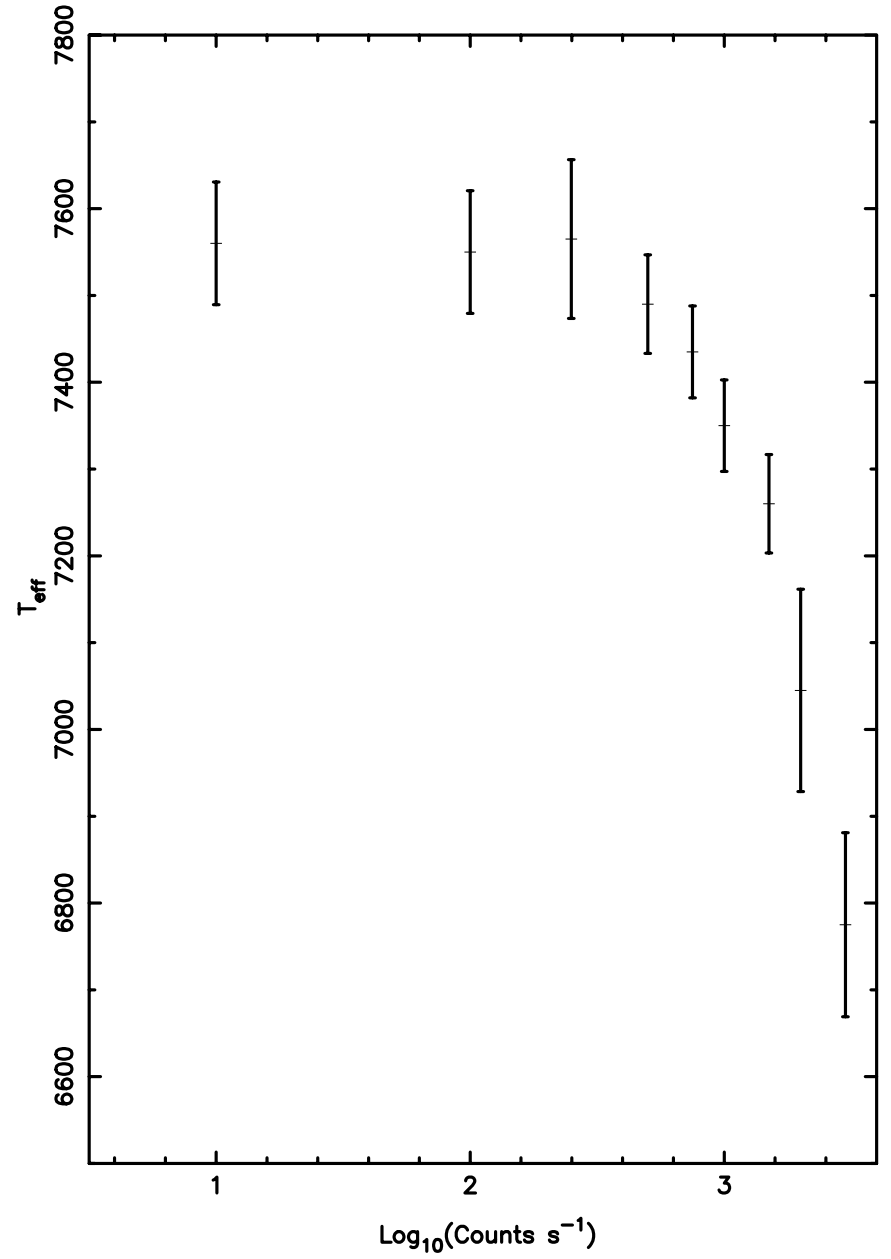

Fig. 4. The predicted variation in measured temperature with count rate, due to distortion of the spectral profile by pile-up. Each data point is derived from the mean of 10 simulations, with $1 \sigma$ error bars. Note that the measured temperature shows no significant drop until count rates exceed $\sim 500 \mathrm{~Hz}$.

or more hotspots on the surface, and/or a bright accretion column. Once this region is occulted by the donor star, however, cooler, fainter components become amenable to study. In the polars these include the rear hemisphere of the donor and the accretion stream itself. In dwarf novae, the disk/stream interaction is seen in addition to the contribution from the secondary. In principle, the stream or disk temperatures can be measured provided the contribution from the secondary is small enough to ignore, or can be subtracted. S-Cam2 is obviously well suited to this kind of study, since the event list data allow spectra to be accumulated across any specified time interval.

\subsection{Stream temperatures in magnetic CVs: HU Aqr, EP Dra, UZ For}

HU Aqr is a polar with a period of $\sim 125$ min. S-Cam 2 observations of this binary are discussed in detail in Bridge et al. (2002b). Three eclipses were observed, each of which was characterised by a very sharp ingress of the bright accretion region, followed by a more gradual ingress of a component identified with the accretion stream. The eclipse with the best $\mathrm{S} / \mathrm{N}$

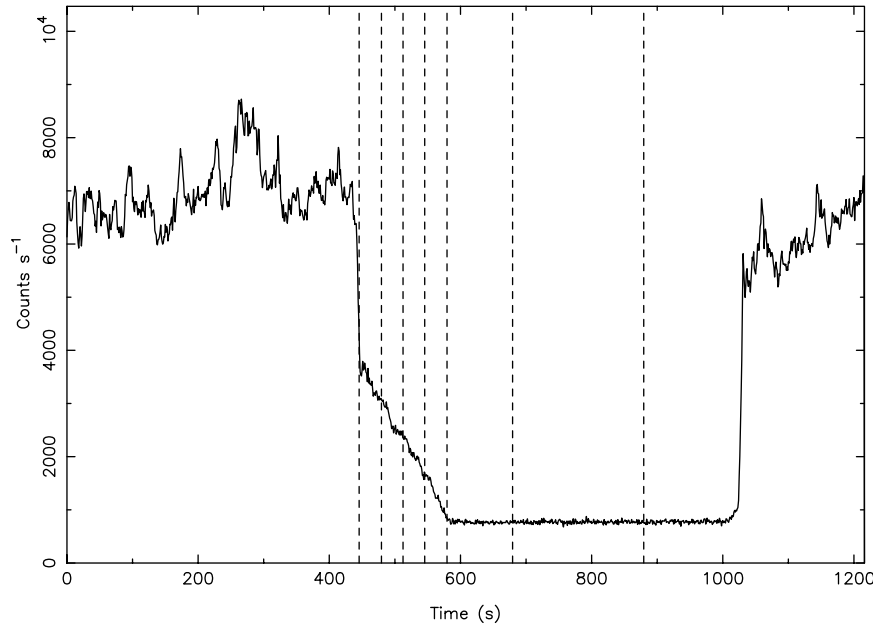

Fig. 5. An eclipse light curve of the polar CV HU Aqr. The vertical bars indicate the eclipse of the accretion stream (divided into four $33 \mathrm{~s}$ components) and the $200 \mathrm{~s}$ portion of the mid-eclipse used for background subtraction.

is shown in Fig. 5, where the accretion stream ingress is very well defined. By studying hardness ratios, Bridge et al. concluded that the light from the system became significantly redder once the accretion region was eclipsed, as expected given that the stream is likely to be much cooler than the accretion region. However the error bars on the data were too large to put any strong constraints on the temperature gradient along the stream.

We have now reexamined the HU Aqr data, this time using the temperature fitting technique. In all three eclipses, a spectrum corresponding to the stream was isolated, together with a portion of mid-eclipse data to use for background subtraction. Using the mid-eclipse as a background has the advantage that we are also subtracting any residual light from the donor star, thereby isolating only the stream.

Of the three eclipses observed by S-Cam2, the first two had insufficient $\mathrm{S} / \mathrm{N}$ (due to seeing, and/or weakness of the feature) to enable the stream to be split into sub-components. The first eclipse yielded $T_{\text {eff }}=6700 \pm 600 \mathrm{~K}$, while the second yielded $T_{\text {eff }}=6500 \pm 500 \mathrm{~K}$. The third eclipse could be split into four time-resolved components, as indicated in Fig. 5. These gavein increasing time order $-T_{\text {eff }}=6600,6600,6700$ and $6300 \mathrm{~K}$, with an estimated error on each measurement of $\pm 600 \mathrm{~K}$. The fit to the first segment of stream data is shown in Fig. 6. Clearly there is no evidence for a gradient in temperature along the stream. Using $U B V R$ photometry, Harrop-Allin et al. (1999) estimated the stream temperature in HU Aqr to lie in the range $6500-7400 \mathrm{~K}$, with $6500 \mathrm{~K}$ being their preferred estimate, in satisfactory agreement with the results reported here. We thus have confidence in extending the technique to other systems.

UZ For and EP Dra are also eclipsing polars which have been the subject of S-Cam2 observations (Perryman et al. 2001; Bridge et al. 2002a). In both cases a stream is sometimes seen, though it is not as well-defined as in HU Aqr. We used the same approach as above, isolating stream/eclipse regions. In the case of EP Dra we observed clear stream ingresses in three eclipses, yielding $T_{\text {eff }}$ values of $5700 \mathrm{~K}, 7200 \mathrm{~K}$ and $6050 \mathrm{~K}$. 


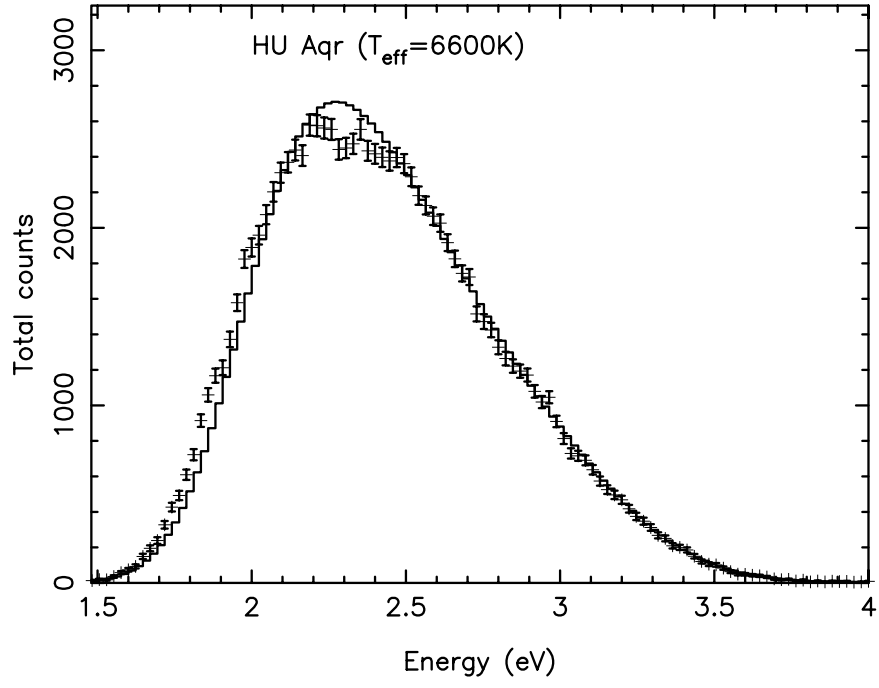

Fig. 6. The black body model fit to the first $33 \mathrm{~s}$ portion of the stream data in HU Aqr. The data has been background subtracted.

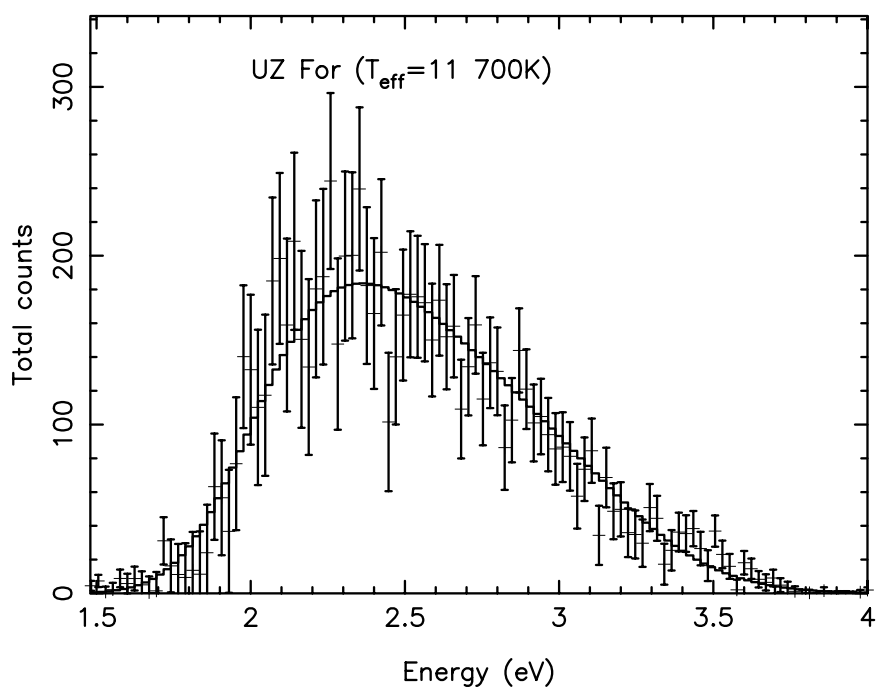

Fig. 7. The black body model fit to the stream component in UZ For. The data has been background subtracted.

The uncertainties were also $\sim \pm 600 \mathrm{~K}$. In this case there is a possible source of systematic error since certain pixels were somewhat unstable during the observations. Clearly, however, the fits point to the stream having a temperature in the same range as that seen in $\mathrm{HU}$ Aqr.

In the case of UZ For there was only one eclipse for which the data quality was sufficiently high to permit the extraction of a stream spectrum. Here, $T_{\text {eff }}$ was found to be $11700 \mathrm{~K} \pm$ $2500 \mathrm{~K}$, significantly higher than for the other two CVs. The fit is shown in Fig. 7. The quoted error includes the uncertainty associated with the model fit, in addition to the uncertain gain (normally our statistics are such that the gain uncertainty is the only significant source of error).

Such a high temperature is not necessarily unreasonable, since the observed temperature of the stream is influenced by a number of processes. The most important are irradiation by X-rays from the white dwarf and magnetic heating in the threading region where material is magnetically confined.
The amount of magnetic heating will be related to the amount and density of material in the stream, and is possibly caused by magnetic reconnection or turbulence (Li 1999) or small shocks (Liebert \& Stockman 1985; Hameury et al. 1986). The amount of irradiative heating of the stream will be dependent upon the area of the stream presented to the white dwarf. The temperature that we determine is then influenced by the area of this heated stream face presented to the observer.

The larger temperature found for UZ For is therefore probably a consequence of observing a larger area of the heated stream face, and stream material that is heated more in the threading region. The UZ For S-Cam2 observations show evidence for two accretion regions (see Perryman et al. 2001). This means that the field lines along which material accretes are filled above and below the orbital plane. We therefore observe more of the heated face of the stream than in HU Aqr or EP Dra where there is evidence for accretion at only one region on the white dwarf (Bridge et al. 2002a, 2002b). Material is still probably heated in HU Aqr at the threading region, as found by Vrielmann \& Schwope (2001) and Harrop-Allin (1999) previously, and this also probably occurs in EP Dra. However, the viewing angle to the stream is such that we do not see as large an area of the heated stream face. Future observations of UZ For will aim to improve the $\mathrm{S} / \mathrm{N}$ of the stream component.

\subsection{The temperature of the white dwarf in the non-magnetic CV IY UMa}

IY UMa is a non-magnetic $\mathrm{CV}$ in which accretion proceeds via a disk. The S-Cam2 observations are discussed in Steeghs et al. (2002). The eclipse morphology is significantly more complex (see Fig. 8, and the discussion in Steeghs et al.). In this case, the geometry precludes easy selection of an accreting region characterised by a single temperature. However it is possible to isolate the spectrum of the white dwarf by extracting the difference between the spectrum of the source immediately before and immediately after the very rapid eclipse of the compact component. Three eclipses were observed by S-Cam2, but the low $\mathrm{S} / \mathrm{N}$ requires the summing of these extracted spectra prior to model fitting. Using a co-added spectrum, therefore, we obtained $T_{\text {eff }}=16000 \pm 3000 \mathrm{~K}$ for the white dwarf in IY UMa (Fig. 9). As in the case of UZ For, the quoted error includes the uncertainty due to the fitting process.

Gänsicke \& Koester (1999) list the effective temperatures for the white dwarfs in the 9 dwarf novae for which the white dwarf spectrum had been unequivocally identified in the UV or optical at the time of their paper. Their list does not include IY UMa. Excluding the long-period system U Gem, which lies above the CV period gap and is significantly hotter than those short-period systems below it, the results span the temperature range $14000-22000 \mathrm{~K}$, with a mean value of $\sim 17000 \mathrm{~K}$. The value we have obtained for IY UMa is therefore quite plausible. Sion (1999) presents a more extensive list of non-magnetic white dwarf temperatures, of which 9 are dwarf novae of the same SU UMa type as IY UMa. Of the 9 objects in Sion's sample, 7 are also found in the Gänsicke \& Koester list, but in most cases the quoted temperatures are somewhat different. 


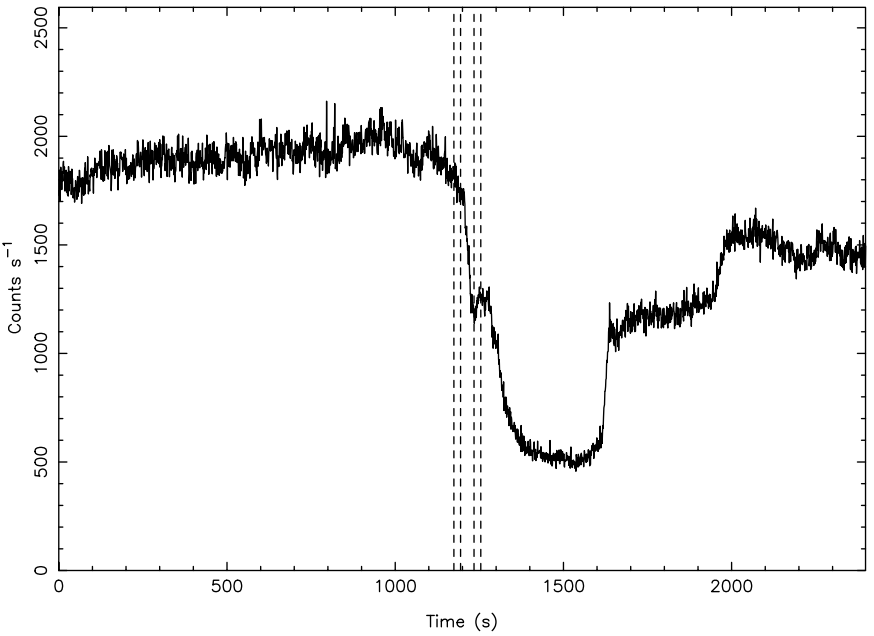

Fig. 8. An eclipse light curve of IY UMa, a non-magnetic CV of the dwarf novae type. The vertical bars indicate the two $20 \mathrm{~s}$ segments of the eclipse used to isolate the spectrum of the white dwarf, immediately before and immediately after the white dwarf ingress.

The temperatures of the Sion sample range from 12000$18500 \mathrm{~K}$, with a mean value of $15600 \mathrm{~K}$. Our value is again consistent with expectations.

\section{Conclusions and future prospects}

In this paper we have outlined a fundamentally new approach to temperature determination using non-dispersive optical spectroscopy. The spectra are modelled with black bodies, modified for atmospheric absorption. For cool stars $\left(T_{\text {eff }}<15000 \mathrm{~K}\right)$, the derived temperatures are generally within $10 \%$ of the literature values. The agreement is less good for hotter stars, but the main sources of error are calibration uncertainties, rather than any intrinsic limitation with the method.

Having demonstrated that the technique works, we have applied it to four cataclysmic binary stars, isolating accretion stream components in three cases and the white dwarf itself in a fourth. The time-tagged photon event list data format of SCam 2 data makes the isolation of these components relatively straightforward. Where appropriate, the results are compared against literature values and found to be in satisfactory agreement.

Work is now underway on the development of S-Cam3, which will incorporate $10 \times 12$ pixels, each of which is slightly larger than those used in S-Cam2. The field of view will therefore be much improved, allowing the edges of the frame to be used for background subtraction with no fear of contamination by source counts. In addition, the energy resolving power is likely to be improved by $20-30 \%$, and realtime monitoring of the gain variations will be possible. All these factors should lead to a significant improvement in the quality of the temperature determination possible with the instrument, in addition to other model-fitting problems such as quasar redshift determination.

Acknowledgements. We thank the other members of the S-Cam2 team for their enthusiastic support throughout the program. In addition,

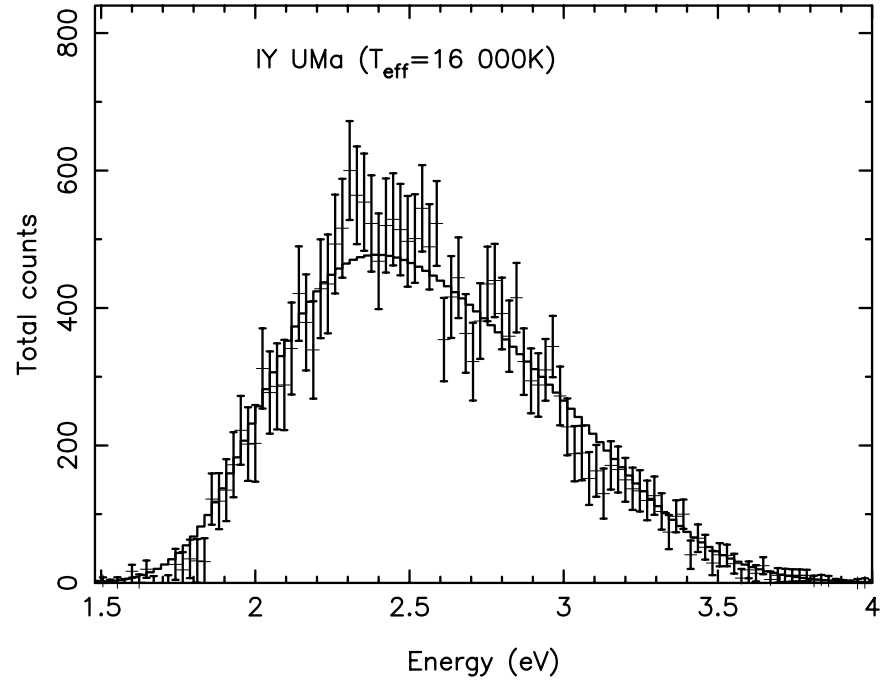

Fig. 9. The black body model fit to the summed spectrum of the white dwarf component in IY UMa. See text for details.

Erik Kuulkers and Danny Steeghs are thanked for useful discussions regarding the $\mathrm{CV}$ temperature fitting. The William Herschel Telescope is operated on the island of La Palma by the Isaac Newton Group in the Spanish Observatoria del Roque de los Muchachos of the Instituto de Astrofisica de Canarias. We thank the anonymous referee for useful comments on this manuscript.

\section{References}

Amado, P. J., \& Byrne, P. B. 1997, A\&A, 319, 967

Arnaud, K. 1996, in Astronomical Data Analysis Software and Systems V, ed. G. Jacoby, \& J. Barnes, ASP Conf. Ser., 101, 17

Bergeron, P., Wesemael, F., Lamontagne, R., et al. 1995, ApJ, 449, 258

Bergeron, P., Leggett, S. K., \& Teresa Ruiz, M. 2001, ApJS, 133, 413

Bridge, C. M., Cropper, M., Ramsay, G., et al. 2002a, MNRAS, 336, 1129

Bridge, C. M., Cropper, M., Ramsay, G., et al. 2002b, MNRAS, submitted

Carramiñana, A., Cadez, A., \& Zwitter, T. 2000, ApJ, 542, 974

de Bruijne, J. H. J., Reynolds, A. P., Perryman, M. A. C., et al. 2002, A\&A, 381, L57

Eikenberry, S. S., Fazio, G. G., Ransom, S. M., et al. 1996, ApJ, 467, L85

Fontaine, G., Brassard, P., Bergeron, P., \& Wesemael, F. 1996, ApJ, 469,320

Gänsicke, \& Koester, D. 1999, A\&A, 346, 151

Greenstein, J. L. 1975, ApJ, 196, L117

Greenstein, J. L. 1976, AJ, 81, 323

Greenstein, J. L. 1984, ApJ, 276, 602

Hameury, J.-M., King, A. R., \& Lasota, J.-P. 1986, MNRAS, 218, 695

Harrop-Allin, M. K. 1999, Ph.D. Thesis, Univ. London

Harrop-Allin, M. K., Cropper, M., Hakala, P. J., Hellier, C., \& Ramseyer, T. 1999, MNRAS, 308, 807

Herbig, G. H. 1999, PASP, 111, 1144

Jacoby, G. H., Hunter, D. A., \& Christian, C. A. 1984, ApJS, 56, 257

Keenan, F. P., \& Dufton, P. L. 1983, MNRAS, 205, 435

Koester, D., Liebert, J., \& Hege, E. K. 1979, A\&A, 71, 163 
Legget, S. K., Allard, F., Berriman, G., et al. 1996, ApJS, 104, 117 Li, J. 1999, in Annapolis Workshop on Magnetic CVs, ed. C. Hellier, \& K. Mukai, ASP Conf. Ser., 157, 235

Liebert, J., \& Stockman, H. S. 1985, in Cataclysmic Variables and Low-Mass X-ray Binaries, ed. D. Q. Lamb, \& J. Patterson (Dordrecht, Reidel), 151

Peacock, A., Verhoeve, P., Rando, N., et al. 1997, A\&AS, 123, 581

Perryman, M. A. C., Foden, C. L., \& Peacock, A. 1993, Nuc. Inst. Meth. A, 325, 319

Perryman, M. A. C., Favata, F., Peacock, A., et al. 1999, A\&A, 346, L30

Perryman, M. A. C., Cropper, M., Ramsay, G., et al. 2001, MNRAS, 324, 899

Rando, N., Peacock, A., Andersson, S., et al. 1998, Proc. SPIE, 3435, 74
Rando, N., Verveer, J., Verhoeve, P., Peacock, A., et al. 2000, Proc. SPIE, ATI-2000, Münich, in press

Robinson, E. L., Mailloux, T. M., Zhang, E., et al. 1995, ApJ, 438, 908

Romani, R. W., Miller, A. J., \& Cabrera, B. 2001, ApJS, 536, 221

Sion, E. M., Greenstein, J. L., Landstreet, J. D., et al. 1983, ApJ, 269, 253

Sion, E. M. 1999, PASP, 111, 532

Steeghs, D., Perryman, M. A. C., Reynolds, A., et al. 2002, MNRAS, in press

Vrielmann, S., \& Schwope, A. D. 2001, MNRAS, 322, 269

Wolff, B., Koester, D., Dreizler, S., \& Haas, S. 1998, A\&A, 329, 1045

Zombeck, M. V. 1990, Handbook of space astronomy and astrophysics, 2nd ed. (Cambridge: Cambridge University Press) 